\title{
USP14/UCHL5 Inhibitor VLX1570
}

National Cancer Institute

\section{Source}

National Cancer Institute. USP14/UCHL5 Inhibitor VLX1570. NCI Thesaurus. Code C121215.

An inhibitor of the 195 proteasome-specific deubiquitylating enzymes (DUBs) USP14 and UCHL5, with apoptosis-inducing and antineoplastic activities. Upon administration, VLX1570 specifically binds to both USP14 and UCHL5, thereby blocking their deubiquitylating activity. This blocks the ubiquitin proteasome degradation pathway, prevents the degradation of defective proteins, and leads to an accumulation of polyubiquitylated proteins. This induces the unfolded protein response (UPR) and results in both the induction of tumor cell apoptosis and the inhibition of tumor cell growth. USP14 and UCHL5, overexpressed in various tumor cell types, play a key role in the correct folding and deubiquitination of proteins. 